\title{
Junction Effect on the Photocatalytic Activity of Mixed-Phase $\mathrm{TiO}_{2}$ Nanoparticles
}

\author{
A. Di Paola, M. Bellardita, L. Palmisano and F. Parrino \\ "Schiavello-Grillone" Photocatalysis Group, Dipartimento di Ingegneria Chimica dei \\ Processi e dei Materiali, Università di Palermo, Viale delle Scienze, 90128 Palermo, Italy. \\ Active $\mathrm{TiO}_{2}$ photocatalysts were prepared under mild experimental \\ conditions by thermohydrolysis of $\mathrm{TiCl}_{4}$ in pure water at $100{ }^{\circ} \mathrm{C}$. \\ The preparation method is very simple and does not require the use \\ of expensive thermal or hydrothermal treatments. Depending on \\ the $\mathrm{TiCl}_{4} / \mathrm{H}_{2} \mathrm{O}$ ratio, pure rutile, binary mixtures of anatase and \\ rutile or anatase and brookite, or ternary mixtures of anatase, \\ brookite and rutile, can be obtained. 4-nitrophenol \\ photodegradation was used to evaluate the photoactivity of the \\ various powders. The high photocatalytic activity of the mixed \\ samples was explained by the presence of junctions among \\ different polymorphic $\mathrm{TiO}_{2}$ phases that allows an improved charge \\ separation of the photogenerated electron-hole pairs, hindering \\ their recombination.
}

\section{Introduction}

$\mathrm{TiO}_{2}$ is the most studied photocatalyst for environmental applications because of its high efficiency, nontoxicity, chemical and biological stability, and low cost (1). $\mathrm{TiO}_{2}$ exists in three different crystalline habits: anatase, brookite and rutile. Anatase is generally accepted to be a photocatalyst more efficient than rutile and brookite but mixtures of the $\mathrm{TiO}_{2}$ polimorphic phases often show photocatalytic activities superior than those of the pure phases (2). The coupling of different semiconductors possessing different energy levels for their corresponding conduction and valence bands is usually beneficial to enhance the photocatalytic activity $(2,3)$. The very active commercial Degussa P25, that is frequently used as a benchmark in heterogeneous photocatalysis, consists of a mixture of anatase and rutile.

The present paper reports on the preparation and characterization of highly active $\mathrm{TiO}_{2}$ photocatalysts prepared by thermohydrolysis of $\mathrm{TiCl}_{4}$ in pure water at $100{ }^{\circ} \mathrm{C}$. The reactivity of the various samples was tested for the photocatalytic degradation of 4nitrophenol and compared to that of Degussa P25. The flat band potential and the band gap values of anatase, brookite and rutile were also determined. The results are discussed by taking account of the relative positions of the energy bands of the three $\mathrm{TiO}_{2}$ phases.

\section{Experimental}

Preparation and characterization of the samples

$1 \mathrm{ml}$ of $\mathrm{TiCl}_{4}$ (Fluka 98\%) was slowly added to different volumes of distilled water at room temperature. The solutions obtained after continuous stirring were heated in closed bottles and aged at $100{ }^{\circ} \mathrm{C}$ in an oven for $48 \mathrm{~h}$. The bottles were allowed to cool and the resultant solids were recovered using a vacuum pump at $55{ }^{\circ} \mathrm{C}$. The crystal phase 
composition of the catalysts was determined by X-ray diffraction (XRD) measurements using a modified Rietveld method (4). The specific surface areas (SSA) were obtained by nitrogen physisorption experiments.

Brookite was separated by peptization from a binary mixture of brookite and rutile, obtained by thermolysis of a solution of $\mathrm{TiCl}_{4}$ in diluted $\mathrm{HCl}\left(\mathrm{TiCl}_{4}: \mathrm{H}_{2} \mathrm{O}: \mathrm{HCl}\right.$ volume ratio 1:300:20) at $100{ }^{\circ} \mathrm{C}$ for $48 \mathrm{~h}(2)$. After repeated washings with water, a dispersion of brookite particles formed while the rutile phase remained as precipitate. The sol containing the brookite particles and the precipitate of rutile were separately dried under vacuum at $55{ }^{\circ} \mathrm{C}$. Anatase was prepared by boiling an aqueous solution of $\mathrm{TiCl}_{4}$ $\left(\mathrm{TiCl}_{4}: \mathrm{H}_{2} \mathrm{O}\right.$ volume ratio 1:50) for $2 \mathrm{~h}$. After removal of the supernatant liquid, the solid was dried under vacuum at $55^{\circ} \mathrm{C}$.

The flat-band potentials of anatase, brookite and rutile were determined by measuring the photovoltage as a function of the suspension $\mathrm{pH}$ in the presence of methyl viologen dichloride (5). The band gap values of the three phases were obtained by diffuse reflectance spectra measurements.

\section{Photoreactivity experiments}

A Pyrex batch photoreactor of cylindrical shape containing $0.5 \mathrm{~L}$ of aqueous suspension was used. A $125 \mathrm{~W}$ medium pressure $\mathrm{Hg}$ lamp (Helios Italquartz, Italy) was immersed within the photoreactor and the photon flux emitted by the lamp was $\Phi_{i}=13.5$ $\mathrm{mW} \cdot \mathrm{cm}^{-2} \cdot \mathrm{O}_{2}$ was continuously bubbled for ca. $0.5 \mathrm{~h}$ before switching on the lamp and throughout the occurrence of the photoreactivity experiments. The amount of catalyst was $0.6 \mathrm{~g} \cdot \mathrm{L}^{-1}$ and the initial 4-nitrophenol (BDH) concentration was $20 \mathrm{mg} \cdot \mathrm{L}^{-1}$. Samples of 5 $\mathrm{mL}$ were withdrawn at fixed intervals of time with a syringe, and the catalyst was separated from the solution by filtration. Some drops of a $1 \mathrm{M} \mathrm{NaOH}$ solution were added before filtration to cause agglomeration of the particles. The quantitative determination of 4-nitrophenol was performed by measuring its absorption at $315 \mathrm{~nm}$ with a spectrophotometer Shimadzu UV-2401 PC.

\section{Results and discussion}

Figure 1 shows the X-ray diffraction patterns of the solids formed by thermohydrolysis of $\mathrm{TiCl}_{4}$ in water at $100{ }^{\circ} \mathrm{C}$. The composition of the powders depended on the $\mathrm{TiCl}_{4} / \mathrm{H}_{2} \mathrm{O}$ ratio and binary or ternary mixtures of the three polimorphs were prevalently produced. The percentages of anatase, brookite and rutile present in the various samples after $48 \mathrm{~h}$ of heating are reported in Table I. The average particle sizes of all the phases present in the various samples were in the range $2-10 \mathrm{~nm}$. 
TABLE I. Influence of the $\mathrm{TiCl}_{4} / \mathrm{H}_{2} \mathrm{O}$ ratio on the relative content (wt\%) of anatase (A), brookite (B) and rutile (R), specific surface area (SSA) and initial reaction rate $\left(\mathrm{r}_{0}\right)$ of the samples.

\begin{tabular}{|c|c|c|c|c|c|c|}
\hline \multirow[t]{2}{*}{ sample } & \multirow{2}{*}{$\begin{array}{c}{\left[\mathrm{TiCl}_{4}\right]} \\
{\left[\mathrm{mol}^{-1} \cdot \mathrm{L}^{-1}\right]}\end{array}$} & \multicolumn{3}{|c|}{ wt \% } & \multirow{2}{*}{$\begin{array}{c}\text { S.S.A } \\
{\left[\mathrm{m}^{2} \cdot \mathrm{g}^{-1}\right]}\end{array}$} & \multirow{2}{*}{$\begin{array}{c}\mathrm{r}_{\mathbf{0}} \cdot \mathbf{1 0}^{9} \\
{\left[\mathrm{~mol} \cdot \mathrm{L}^{-1} \cdot \mathrm{s}^{-1}\right]}\end{array}$} \\
\hline & & A & B & $\mathbf{R}$ & & \\
\hline $\mathrm{TiO}_{2}(1: 5)$ & 1.48 & & & 100 & 87.5 & 31 \\
\hline $\mathrm{TiO}_{2}(1: 10)$ & 0.81 & 19 & & 81 & 108.0 & 63 \\
\hline $\mathrm{TiO}_{2}(1: 25)$ & 0.34 & 53 & & 47 & 201.5 & 61 \\
\hline $\mathrm{TiO}_{2}(1: 35)$ & 0.25 & 70 & 19 & 11 & 196.1 & 47 \\
\hline $\mathrm{TiO}_{2}(1: 50)$ & 0.17 & 66 & 21 & 13 & 196.6 & 58 \\
\hline $\mathrm{TiO}_{2}(1: 75)$ & 0.12 & 65 & 28 & 7 & 216.1 & 76 \\
\hline $\mathrm{TiO}_{2}(1: 100)$ & 0.09 & 66 & 28 & 6 & 219.1 & 67 \\
\hline $\mathrm{TiO}_{2}(1: 150)$ & 0.06 & 77 & 23 & & 208.3 & 33 \\
\hline
\end{tabular}

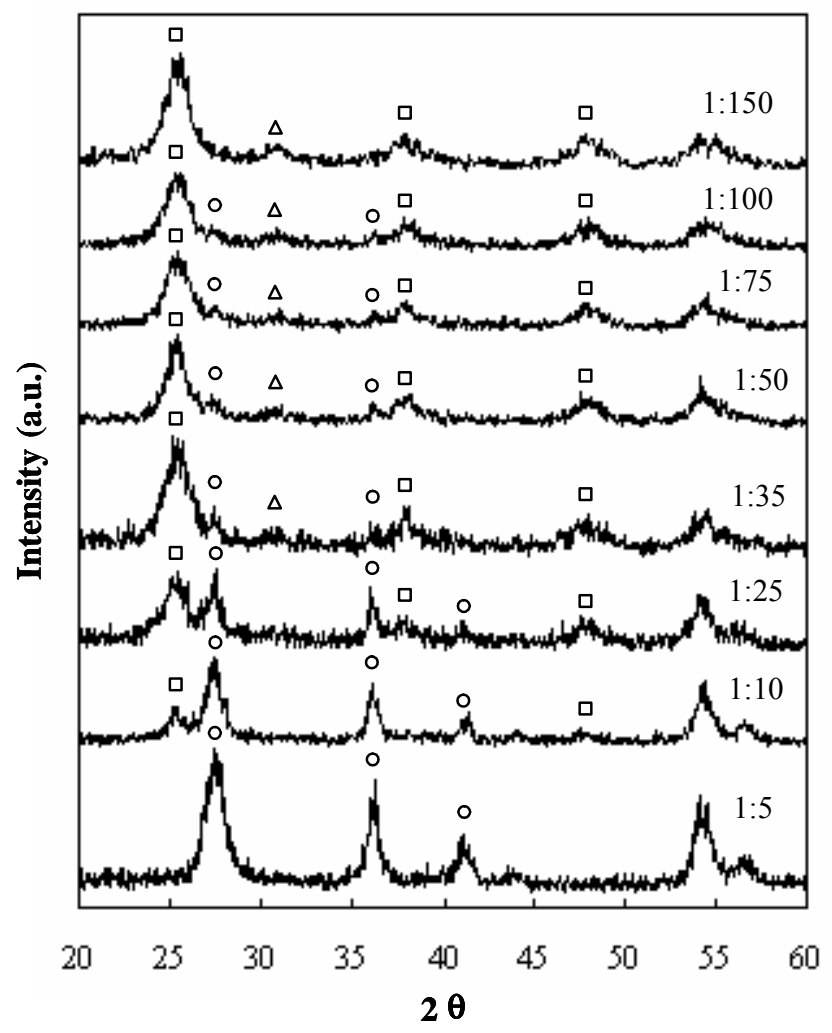

Figure 1. XRD patterns of the solids obtained by thermohydrolysis of $\mathrm{TiCl}_{4}$ in water at $100{ }^{\circ} \mathrm{C}$ for 48 h. ( $\square$ ) anatase; (O) rutile; $(\Delta)$ brookite.

The $\mathrm{H}_{2} \mathrm{O}$ content is the factor which determines the nature of the crystalline phases and their relative proportions. The fraction of rutile decreases with dilution and this is consistent with the results of previous works indicating that low $\mathrm{pH}$ values favour the formation of rutile $(6,7)$. Only rutile was obtained when the $\mathrm{TiCl}_{4} / \mathrm{H}_{2} \mathrm{O}$ ratio was 1:5. The $\mathrm{TiCl}_{4} / \mathrm{H}_{2} \mathrm{O}$ ratio plays an important role in the competition between the formation of anatase and rutile, but has little effect on brookite formation.

Figure 2 shows a microscopic procedure proposed to explain the formation of the different $\mathrm{TiO}_{2}$ polymorphic species. According to Zheng et al. a low concentration $\mathrm{TiCl}_{4}$ solution contains a large amount of disperse $\left[\mathrm{Ti}(\mathrm{OH})_{2}\left(\mathrm{OH}_{2}\right)_{4}\right]^{2+}$ octahedral complexes (8). As a consequence of hydrothermic treatments, the octahedra link together by olation, 
through dehydration reactions between aquo and hydroxo ligands. Rutile type nuclei are developed if the $\left[\mathrm{Ti}(\mathrm{OH})_{2}\left(\mathrm{OH}_{2}\right)_{4}\right]^{2+}$ monomers combine by sharing equatorial edges, whereas anatase or brookite type nuclei form if the monomers combine by sharing apical edges. Further growth proceeds by formation of linear chains from the rutile type nuclei or of skewed chains from the anatase or brookite type nuclei. All the three polymorphic phases can be obtained contemporaneously and the composition of the mixtures depends on the $\mathrm{TiCl}_{4} / \mathrm{H}_{2} \mathrm{O}$ ratio and by the reaction time.

When the concentration of $\mathrm{TiCl}_{4}$ is high, the solution should prevalently contain $\left[\mathrm{TiO}\left(\mathrm{OH}_{2}\right)_{5}\right]^{2+}$ momomers that can combine by olation only by sharing equatorial edges. In this case, rutile crystallites are developed (8).

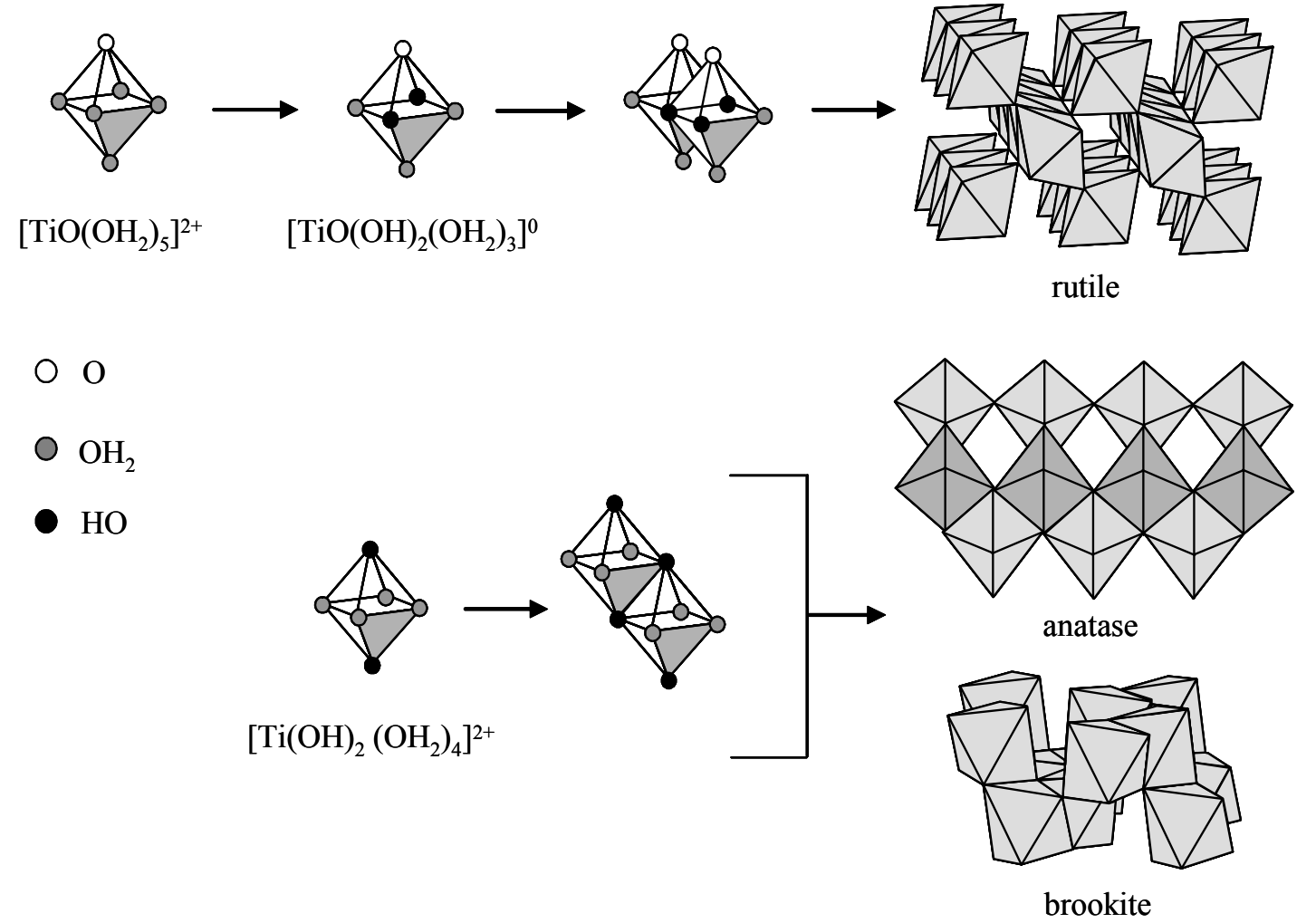

Figure 2. Possible reaction pathways for the formation of rutile, anatase and brookite starting from octahedral complexes: $\left[\mathrm{TiO}(\mathrm{OH})_{2}\left(\mathrm{OH}_{2}\right)_{3}\right]^{0}$ and $\left[\mathrm{Ti}(\mathrm{OH})_{2}\left(\mathrm{OH}_{2}\right)_{4}\right]^{2+}(8)$.

The photocatalytic activity of the samples, as well their composition, depends on the $\mathrm{TiCl}_{4} / \mathrm{H}_{2} \mathrm{O}$ ratio. Table I reports the values of the initial degradation rate of 4-nitrophenol, $\mathrm{r}_{0}$, calculated from the initial slope of the concentration versus time profiles. The mixed systems revealed an enhanced photoactivity compared with that of the pure $\mathrm{TiO}_{2}$ polymorphic phases and some samples were more active than Degussa P25. The most efficient samples consisted of a ternary mixture of anatase, brookite and rutile.

The high photocatalytic activity of the binary or ternary mixtures can be explained by the presence of junctions among different polymorphic $\mathrm{TiO}_{2}$ phases possessing different energy levels for their corresponding conduction and valence bands (3). A current hypothesis of the enhanced activity of mixed phases is the vectorial transfer of electrons 
from a semiconductor to another, leading to more efficient electron-hole separation and greater catalytic reactivity (9).

Diffuse reflectance measurements allowed to determine the band gap of pure anatase, brookite and rutile. The estimated values were $3.05 \mathrm{eV}$ for anatase, $3.26 \mathrm{eV}$ for brookite and $2.98 \mathrm{eV}$ for rutile, respectively. The band gaps of the mixtures of two or three phases were lower than those of anatase and brookite and near to that of rutile.

The values of the flat-band potentials of the three pure phases were determined by the slurry method proposed by Roy et al. (5), measuring the variation of the photovoltage with the $\mathrm{pH}$ of suspensions of the powders in the presence of an electron acceptor. Figure 3 shows the effect of $\mathrm{pH}$ on the photovoltage developed on irradiation of anatase, brookite or rutile suspensions. From the value of the inflection point $\left(\mathrm{pH}_{0}\right)$, the flat band potential at $\mathrm{pH} 7$ was calculated by the equation:

$$
\mathrm{E}_{\mathrm{FB}}(\mathrm{pH}=7)=\mathrm{E}_{\mathrm{MV}}{ }^{2+/+}+0.059\left(\mathrm{pH}_{0}-7\right)
$$

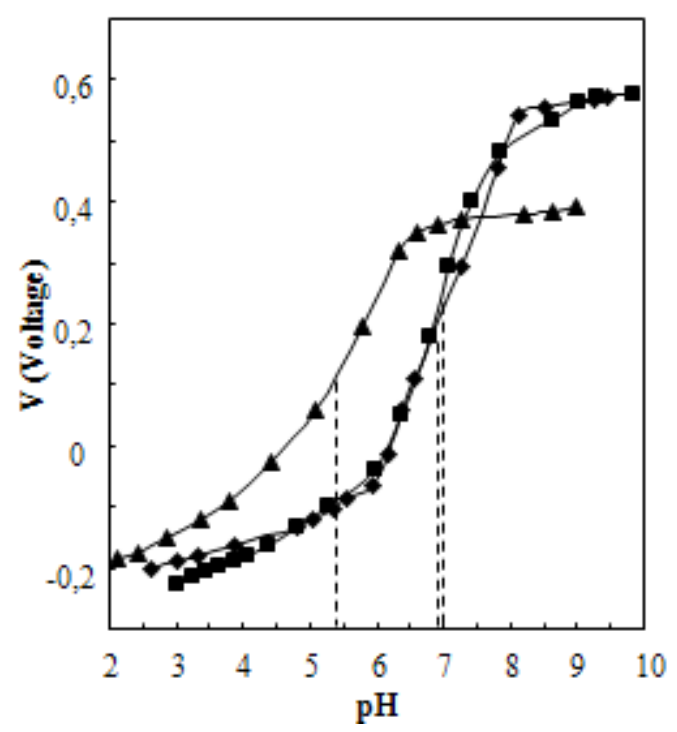

Figure 3. Effect of $\mathrm{pH}$ on the photovoltage developed on irradiation of ( $\bullet$ anatase, ( $\bullet$ ) brookite and $(\boldsymbol{\Lambda})$ rutile suspensions in the presence of methyl viologen dichloride.

The values obtained were $-0.45 \mathrm{~V},-0.46 \mathrm{~V}$ and $-0.37 \mathrm{~V}$ for anatase, brookite and rutile, respectively.

Assuming that the difference between flat band potential and conduction band edge is negligible, it is possible to locate the valence band edge of the three semiconductors by adding the band gap energy to the flat-band potential value. Figure 4 shows the relative positions of the energy bands of anatase, brookite and rutile, at $\mathrm{pH}=7$. Anatase and brookite differ in the position of their valence band since brookite has a slightly larger band gap energy. 


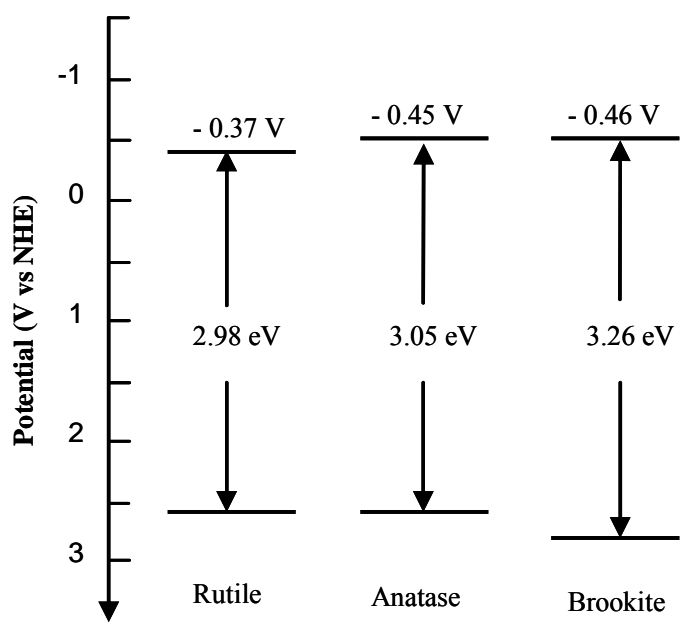

Figure 4. Electrochemical potentials (versus NHE) of the band edges of anatase, brookite, and rutile at $\mathrm{pH}=7$.

From the relative position of the band edges, it can be expected that electrons pass from the more cathodic conduction band of one semiconductor to the more anodic conduction band of another component. Another explanation recently proposed by Hurum et al. (9) that cannot be excluded is that electron transfer occurs from the more anodic conduction band of one phase (e.g. rutile) to trapping sites of another polymorph (e.g. anatase) which are lower in energy than the conduction band.

The photoactivity of the mixed powders depends on the composition, the aggregates size and the distribution of the various junctions among different phases. The small size of the particles and the intimate contact with each other are crucial factors to increase the efficiency of the samples.

\section{Conclusion}

Highly active photocatalytic $\mathrm{TiO}_{2}$ samples can be synthesized by thermohydrolysis of $\mathrm{TiCl}_{4}$ in water at $100{ }^{\circ} \mathrm{C}$. The most efficient samples consisted of a ternary mixture of anatase, brookite and rutile. The presence of junctions among different polymorphic $\mathrm{TiO}_{2}$ phases enhances the separation of the photogenerated electron-hole pairs, reducing their recombination.

The composition of the samples can be easily tailored by simply varying the $\mathrm{TiCl}_{4} / \mathrm{H}_{2} \mathrm{O}$ volume ratio. A proper $\mathrm{TiCl}_{4} / \mathrm{H}_{2} \mathrm{O}$ volume ratio is important for the synthesis of a ternary mixture of anatase, brookite and rutile which exhibits the highest photoactivity.

\section{Acknowledgments}

This work was financially supported by the Ministero dell'Istruzione, dell'Università e della Ricerca (Roma). 


\section{References}

1. A. Fujishima, K. Hashimoto and T. Watanabe, $\mathrm{TiO}_{2}$ Photocatalysis: Fundamentals and Applications, Bkc, Tokyo (1999).

2. A. Di Paola, G. Cufalo, M. Addamo, M. Bellardita, R. Campostrini, R. Ceccato, M. Ischia and L. Palmisano, Colloid. Surf. A: Physicochem. Eng. Aspects, 317, 366 (2008).

3. N. Serpone, P. Maruthamuthu, P. Pichat, E. Pelizzetti and H. Hidaka, J. Photochem. Photobiol. A: Chem., 85, 247 (1995).

4. L. Lutterotti, R. Ceccato, R. Dal Maschio and E. Pagani, Mater. Sci. Forum, 278281, 87 (1998).

5. A. M. Roy, G. C. De, N. Sasmal and S. S. Bhattacharyya, Int. J. Hydrogen Energy, 20, 627 (1995).

6. A. Pottier, C. Chanéac, E. Tronc, L. Mazerolles and J.-P. Jolivet, J. Mater. Chem. 11, 1116 (2001).

7. J. H. Lee and Y. S. Yang, Mater. Chem. Phys., 93, 237 (2005).

8. Y. Zheng, E. Shi, Z. Chen, W. Li and X. Hu, J. Mater. Chem. 11, 1547 (2001).

9. D. C. Hurum, A. G. Agrios, K. A. Gray, T. Rajh and M. C. Thurnauer, J. Phys. Chem. B, 107, 4545 (2003). 